\title{
The Extreme and Support Points of a New Class of Analytic Functions with Positive Real Part
}

\author{
Zhigang Peng \\ Faculty of Mathematics and Computer Science, Hubei University, Wuhan, Hubei 430062, China \\ Correspondence should be addressed to Zhigang Peng; pengzhigang65@yahoo.com.cn
}

Received 10 October 2012; Accepted 13 December 2012

Academic Editor: Jacek Dziok

Copyright (c) 2013 Zhigang Peng. This is an open access article distributed under the Creative Commons Attribution License, which permits unrestricted use, distribution, and reproduction in any medium, provided the original work is properly cited.

Suppose that $0<\alpha<\beta<+\infty$. Let $\mathscr{P}(\alpha, \beta)$ denote the set of functions $p(z)$ that are analytic in $D=\{z:|z|<1\}$ and satisfy $\operatorname{Re} p(z)>0(|z|<1)$ and $\alpha \leq p(0) \leq \beta$. In this paper, we investigate the extreme points and support points of $\mathscr{P}(\alpha, \beta)$.

\section{Introduction}

By $\mathscr{A}$, we denote the space of functions analytic in the unit disk $D=\{z:|z|<1\}$. Endowed with the topology of uniform convergence on compact subsets of the unit disk, $\mathscr{A}$ is a locally convex topological vector space.

Let $X$ be a topological vector space and $U$ a subset of $X$. If $V \subset U$, then $V$ is called an extremal subset of $U$ provided that whenever $u=t x+(1-t) y$, where $u \in V, x, y \in U$ and $0<t<1$, then $x$ and $y$ both belong to $V$. An extremal subset of $U$ consisting of just one point is called an extreme point of $U$. Thus, an element $x \in U$ is an extreme point of $U$ if and only if $x$ is not a proper convex combination of any two distinct points in $U$. The set of all extreme points of $U$ is denoted by $E U$. It is apparent that if $V$ is an extremal subset of $U$, then $E V \subset E U$. If $X$ is a locally convex topological vector space and $U$ is a nonempty compact subset of $X$, then $E U$ is nonempty [1, page 44], [2, page 181]. For any subset $U$ of $X$, we use $H U$ to denote the closed convex hull of $U$. If $U$ is a compact subset of the locally convex topological vector space $X$, then, by Krein-Milman theorem [1, page 44], [2, page 182], $H U=H E U$.

Let $\mathscr{P}$ be the set of all functions $p(z)$ which are analytic, have positive real part in $D$, and satisfy $p(0)=1$. Then $\mathscr{P}$ is a compact subset of $\mathscr{A}[1$, page 39]. It is well known that $E \mathscr{P}=\{(1+x z) /(1-x z):|x|=1\}[1$, page 48], [3-5]. Bellamy and Tkaczyńska [6] investigated the extreme points of some classes of analytic functions with positive real part and a prescribed set of coefficients. Peng [7] investigated the extreme points of a class of analytic functions with positive real part and a prescribed set of values.

Suppose that $\mathscr{F}$ is a compact subset of $\mathscr{A}$. A function $f$ is called a support point of $\mathscr{F}$ if $f \in \mathscr{F}$ and there is a continuous linear functional $J$ on $\mathscr{A}$ such that $\operatorname{Re} J$ is nonconstant on $\mathscr{F}$ and

$$
\operatorname{Re} J(f)=\max \{\operatorname{Re} J(g): g \in \mathscr{F}\} .
$$

The set of all support points of $\mathscr{F}$ is denoted by supp $\mathscr{F}$. Hallenbeck and MacGregor [1, page 94], [8] proved that the set supp $\mathscr{P}$ consists of all functions which may be written as

$$
p(z)=\sum_{k=1}^{m} \lambda_{k} \frac{1+x_{k} z}{1-x_{k} z}
$$

where $\lambda_{k} \geq 0, \sum_{k=1}^{m} \lambda_{k}=1$, and $\left|x_{k}\right|=1(m=1,2, \ldots)$. The author [9] investigated the support points of a class of analytic functions with positive real part and a prescribed set of coefficients.

Suppose that $0<\alpha<\beta<+\infty$. Let $\mathscr{P}(\alpha, \beta)$ denote the set of functions $p(z)$ that are analytic in $D=\{z:|z|<1\}$ and satisfy $\operatorname{Re} p(z)>0(|z|<1)$ and $\alpha \leq p(0) \leq \beta$. It is apparent that $p(z) \in \mathscr{P}(\alpha, \beta)$ if and only if $p(z)=\mu q(z)$ with some $q(z) \in \mathscr{P}$ and $\alpha \leq \mu \leq \beta$. Thus, it is easy to prove that $\mathscr{P}(\alpha, \beta)$ is a compact subset of $\mathscr{A}$. In this paper we investigate the extreme points and support points of $\mathscr{P}(\alpha, \beta)$. In some ways, the results we obtained generalize the results of Holland, Hallenbeck, and MacGregor. 


\section{Main Results}

Theorem 1. $p(z)$ is an extreme point of $\mathscr{P}(\alpha, \beta)$ if and only if

$$
p(z)=\alpha \frac{1+x z}{1-x z}, \quad \text { or } \quad \beta \frac{1+x z}{1-x z},
$$

where $|x|=1$.

Proof. Suppose that

$$
\alpha \frac{1+x z}{1-x z}=t p_{1}(z)+(1-t) p_{2}(z)
$$

where $|x|=1,0<t<1, p_{1}, p_{2} \in \mathscr{P}(\alpha, \beta)$. Then $\alpha=t p_{1}(0)+$ $(1-t) p_{2}(0)$. Since $\alpha \leq p_{1}(0), \alpha \leq p_{2}(0)$, it follows that $p_{1}(0)=$ $p_{2}(0)=\alpha$. Consequently, $p_{1}(z) / \alpha \in \mathscr{P}, p_{2}(z) / \alpha \in \mathscr{P}$. Notice that (4) is equivalent to

$$
\frac{1+x z}{1-x z}=t \frac{1}{\alpha} p_{1}(z)+(1-t) \frac{1}{\alpha} p_{2}(z),
$$

and that $[1+x z] /[1-x z] \in E \mathscr{P}$, and we have

$$
\frac{1}{\alpha} p_{1}(z)=\frac{1}{\alpha} p_{2}(z) .
$$

So, $p_{1}(z)=p_{2}(z)$. This proves that $[\alpha(1+x z)] /[1-x z] \epsilon$ $E \mathscr{P}(\alpha, \beta)$. Similarly, we can prove that $[\beta(1+x z)] /[1-x z] \epsilon$ $E \mathscr{P}(\alpha, \beta)$.

Conversely, Suppose $p(z) \in E \mathscr{P}(\alpha, \beta)$. If $\alpha<p(0)<\beta$, then $p(0)=t \alpha+(1-t) \beta$ for some $0<t<1$. Since

$$
\begin{gathered}
p(z)=t \frac{\alpha}{p(0)} p(z)+(1-t) \frac{\beta}{p(0)} p(z), \\
\frac{[\alpha p(z)]}{p(0)} \in \mathscr{P}(\alpha, \beta), \quad \frac{[\beta p(z)]}{p(0)} \in \mathscr{P}(\alpha, \beta), \\
\frac{[\alpha p(z)]}{p(0)} \neq \frac{[\beta p(z)]}{p(0)},
\end{gathered}
$$

it follows that $p(z) \notin E \mathscr{P}(\alpha, \beta)$ which contradicts the assumption. Thus $p(0)=\alpha$ or $p(0)=\beta$.

If $p(0)=\alpha$, then $p(z) / \alpha \in \mathscr{P}$. By Herglotz formula [ 1 , page 30$]$, [10, page 22$]$, we have

$$
p(z)=\alpha \int_{|x|=1} \frac{1+x z}{1-x z} d \mu(x),
$$

where $\mu$ is a probability measure on the unit circle $|x|=1$. If $\mu$ is not a point mass, then there exist probability measures $\mu_{1}$ and $\mu_{2}$ on the unit circle $|x|=1$ such that $\mu_{1} \neq \mu_{2}$ and $\mu=t \mu_{1}+(1-t) \mu_{2}$ for some $t$ with $0<t<1$ [1, page 47]. Let

$$
\begin{aligned}
& p_{1}(z)=\alpha \int_{|x|=1} \frac{1+x z}{1-x z} d \mu_{1}(x), \\
& p_{2}(z)=\alpha \int_{|x|=1} \frac{1+x z}{1-x z} d \mu_{2}(x) .
\end{aligned}
$$

Then $p_{1}, p_{2} \in \mathscr{P}(\alpha, \beta), p_{1} \neq p_{2}$, and $p=t p_{1}+(1-t) p_{2}$. This implies that $p$ is not an extreme point of $\mathscr{P}(\alpha, \beta)$. We get a contradiction. So, $\mu$ is a point mass. Without loss of generality, we suppose $\mu(\{x\})=1$ for some unit complex number $x$. Then $p(z)=\alpha(1+x z) /(1-x z)$ with $|x|=1$. Similarly, if $p(0)=\beta$, then we can prove that $p(z)=\beta(1+x z) /(1-x z)$, where $|x|=1$. The proof is completed.

Theorem 2. $p$ is a support point of $\mathscr{P}(\alpha, \beta)$ if and only if $p$ satisfies one of the following conditions:

(1) $p \in \mathscr{P}(\alpha, \beta), p(0)=\alpha$ or $\beta$,

(2)

$$
p(z)=\alpha \sum_{k=1}^{m} \lambda_{k} \frac{1+x_{k} z}{1-x_{k} z}+\beta \sum_{k=m+1}^{m+n} \lambda_{k} \frac{1+x_{k} z}{1-x_{k} z},
$$

where $\lambda_{k} \geq 0, \sum_{k=1}^{m+n} \lambda_{k}=1,\left|x_{k}\right|=1, m$ and $n$ are positive integers.

Proof. Let $p_{0}$ be a support point of $\mathscr{P}(\alpha, \beta)$. Then there is a continuous linear functional $J$ defined on $\mathscr{A}$ such that $\operatorname{Re} J$ is not constant on $\mathscr{P}(\alpha, \beta)$ and

$$
\operatorname{Re} J\left(p_{0}\right)=\max \{\operatorname{Re} J(p): p \in \mathscr{P}(\alpha, \beta)\} .
$$

Suppose that $\operatorname{Re} J\left(p_{0}\right)=M$. Let $\mathbb{Q}=\{q: q \in \mathscr{P}(\alpha, \beta)$, $\operatorname{Re} J(q)=M$ \}. Since $p_{0} \in \mathbb{Q}$ and $J$ is continuous, $\mathbb{Q}$ is a nonvacuous closed convex subset of $\mathscr{P}(\alpha, \beta)$. As $\mathscr{P}(\alpha, \beta)$ is a compact subset of $\mathscr{A}$, so is $\mathbb{Q}$. By Krein-Milman theorem [1, page 44], [2, page 182], EQ is nonempty, and $Q=H E Q$. Suppose that $q \in \mathbb{Q}$ and $q=t q_{1}+(1-t) q_{2}$, where $q_{1}$, $q_{2} \in \mathscr{P}(\alpha, \beta)$ and $0<t<1$. Then

$$
\begin{aligned}
M=\operatorname{Re} J(q) & =t \operatorname{Re} J\left(q_{1}\right)+(1-t) \operatorname{Re} J\left(q_{2}\right) \\
& \leq t M+(1-t) M=M .
\end{aligned}
$$

Since $\operatorname{Re} J\left(q_{1}\right) \leq M, \operatorname{Re} J\left(q_{2}\right) \leq M$, it follows from (12) that

$$
\operatorname{Re} J\left(q_{1}\right)=\operatorname{Re} J\left(q_{2}\right)=M \text {. }
$$

This implies that $q_{1}, q_{2} \in \mathbb{Q}$. Thus $Q$ is an extremal subset of $\mathscr{P}(\alpha, \beta)$ and so $E \mathbb{Q} \subset E \mathscr{P}(\alpha, \beta)$.

Since $J$ is a continuous linear functional on $\mathscr{A}$, there is a sequence $\left\{b_{n}\right\}$ of complex numbers satisfying

$$
\varlimsup_{n \rightarrow \infty}\left(\left|b_{n}\right|\right)^{1 / n}<1,
$$

such that

$$
J(f)=\sum_{n=0}^{\infty} b_{n} a_{n}
$$

where $f \in \mathscr{A}$ and $f(z)=\sum_{n=0}^{\infty} a_{n} z^{n}$ [1, page 42].

Let

$$
G(x)=J\left(\frac{1+x z}{1-x z}\right)=b_{0}+\sum_{n=1}^{\infty} 2 b_{n} x^{n} .
$$

Then $G(x)$ is analytic in $\bar{D}=\{x:|x| \leq 1\}$. 
If $E Q$ has infinitely many elements, then there must be infinitely many $[\alpha(1+x z)] /(1-x z)$ or infinitely many $[\beta(1+$ $x z)] /(1-x z)$ in $E Q$, where $|x|=1$. Without loss of generality, we assume that there are infinitely many $[\alpha(1+x z)] /(1-x z)$ in $E Q$ with $|x|=1$. Then $\operatorname{Re} G(x)=M / \alpha$ has infinitely many solutions which implies that $G(x)$ is constant in $\bar{D}=\{z$ : $|z| \leq 1\}$. So, $E Q$ contains all $[\alpha(1+x z)] /(1-x z)$ with $|x|=1$ but contains no elements such as $[\beta(1+x z)] /(1-x z)$ with $|x|=1$. This prove that

$$
E \mathbb{Q}=\left\{\frac{[\alpha(1+x z)]}{1-x z}:|x|=1\right\},
$$

and thus

$$
\mathbb{Q}=H E \mathbb{Q}=\{p: p \in \mathscr{P}(\alpha, \beta), p(0)=\alpha\} .
$$

In particular, $p_{0} \in \mathscr{P}(\alpha, \beta)$ and $p_{0}(0)=\alpha$. Similarly, if we assume that there are infinitely many $[\beta(1+x z)] /(1-x z)$ in $E Q$ with $|x|=1$, then we can prove that $p_{0} \in \mathscr{P}(\alpha, \beta)$ and $p_{0}(0)=\beta$.

In the case that $E Q$ has only a finite number of elements, say, $E Q=\left\{\left[\alpha\left(1+x_{k} z\right)\right] /\left(1-x_{k} z\right),\left[\beta\left(1+x_{j} z\right)\right] /\left(1-x_{j} z\right)\right.$ : $\left.\left|x_{k}\right|=\left|x_{j}\right|=1, k=1, \ldots, m ; j=1, \ldots, n\right\}$. Then $\mathbb{Q}=H E \mathbb{Q}$ consists of functions given by (10). $p_{0}$ especially must have the form given by (10).

Conversely, Suppose that $p_{0} \in \mathscr{P}(\alpha, \beta)$ and that $p_{0}(0)=$ $\alpha$. Define a continuous linear functional $J$ on $\mathscr{A}$ by $J(f)=-a_{0}$ where $f(z)=\sum_{n=0}^{\infty} a_{n} z^{n}$. Since $\alpha<\beta$, it is clear that Re $J$ is not constant on $\mathscr{P}(\alpha, \beta)$ and $\operatorname{Re} J\left(p_{0}\right)=\max \{\operatorname{Re} J(p): p \epsilon$ $\mathscr{P}(\alpha, \beta)\}$. So, $p_{0} \in \operatorname{supp} \mathscr{P}(\alpha, \beta)$. Similarly, we can prove that $p_{0} \in \operatorname{supp} \mathscr{P}(\alpha, \beta)$ if $p \in \mathscr{P}(\alpha, \beta)$ and $p(0)=\beta$.

Now suppose that $p_{0}$ has the form (10). Then, by Lemma 7.2 in [1], there is a function $F$ analytic on $\bar{D}=\{z$ : $|z| \leq 1\}$ such that $\operatorname{Re} F(z) \leq 0$ when $|z| \leq 1$ and $\operatorname{Re} F(z)=0$ if and only if $z=x_{k}(k=1,2, \ldots, m+n)$. Suppose that $F(z)=\sum_{n=0}^{\infty} d_{n} z^{n}$. Let

$$
b_{0}=d_{0}, \quad b_{n}=\frac{d_{n}}{2} \quad(n=1,2, \ldots) .
$$

Then $\varlimsup_{n \rightarrow \infty}\left|b_{n}\right|^{1 / n}<1$. Define a linear functional $J$ on $\mathscr{A}$ by

$$
J(f)=\sum_{n=0}^{\infty} b_{n} a_{n}
$$

where

$$
f(z)=\sum_{n=0}^{\infty} a_{n} z^{n} \in \mathscr{A} .
$$

Then $J$ is continuous [1, page 42]. Since

$$
\begin{aligned}
& J\left(\frac{\alpha(1+x z)}{(1-x z)}\right)=\alpha F(x), \\
& J\left(\frac{\beta(1+x z)}{(1-x z)}\right)=\beta F(x),
\end{aligned}
$$

it follows that [1, page 44]

$$
\begin{aligned}
\max & \{\operatorname{Re} J(p): p \in \mathscr{P}(\alpha, \beta)\} \\
& =\max \{\operatorname{Re} J(p): p \in E \mathscr{P}(\alpha, \beta)\}=0 .
\end{aligned}
$$

Note that

$$
\begin{array}{r}
\operatorname{Re} J\left(\frac{\left(1+x_{k} z\right)}{\left(1-x_{k} z\right)}\right)=\operatorname{Re} F\left(x_{k}\right)=0 \\
(k=1,2, \ldots, m+n),
\end{array}
$$

we have $\operatorname{Re} J\left(p_{0}\right)=0$. If $\operatorname{Re} J$ is constant on $\mathscr{P}(\alpha, \beta)$, then $\operatorname{Re} F(x)=0$ when $|x|=1$. But it is not the case. Therefore $p_{0} \in \operatorname{supp} \mathscr{P}(\alpha, \beta)$.

Remark 3. Though we assume that $\alpha<\beta$ in Theorem 1, it is easy to see that Theorem 1 is valid for $\alpha=\beta=1$, which is just the result of Holland. For $\alpha=\beta=1$, Theorem 2 is invalid, since $p \in \mathscr{P}(1,1)=\mathscr{P}$ and $p(0)=1$ does not imply that $p$ is a support point of $\mathscr{P}$. In the case where $\alpha=\beta=1$, Theorem 2 should be stated as follows.

The set supp $\mathscr{P}$ consists of all functions which may be written as

$$
p(z)=\sum_{k=1}^{m} \lambda_{k} \frac{1+x_{k} z}{1-x_{k} z}
$$

where $\lambda_{k} \geq 0, \sum_{k=1}^{m} \lambda_{k}=1$, and $\left|x_{k}\right|=1(m=1,2, \ldots)$.

This is the result of Hallenbeck and MacGregor [1, page 94], [8]. It is easy to see that Theorem 2 generalizes the result of Hallenbeck and MacGregor in some sense.

\section{Acknowledgments}

The author expresses his heartfelt thanks to the referee for his (or her) critical review and helpful suggestions for the improvement of this paper. This paper is supported by the Educational Commission of Hubei Province of China (D2011006).

\section{References}

[1] D. J. Hallenbeck and T. H. MacGregor, Linear Problems and Convexity Techniques in Geometric Function Theory, Pitman Advanced Publishing Program, Boston, Mass, USA, 1984.

[2] A. E. Taylor and D. C. Lay, Introduction to Functional Analysis, John Wiley \& Sons, New York, NY, USA, 2nd edition, 1980.

[3] J. G. Clunie, "Some remarks on extreme points in function theory," in Aspects of Contemporary Complex Analysis, pp. 137-146, Academic Press, London, UK, 1980.

[4] R. A. Kortram, "The extreme points of a class of functions with positive real part," Bulletin of the Belgian Mathematical Society, vol. 4, no. 4, pp. 449-459, 1997.

[5] F. Holland, "The extreme points of a class of functions with positive real part," Mathematische Annalen, vol. 202, pp. 85-87, 1973.

[6] D. P. Bellamy and K. Tkaczyńska, "Extreme points of some classes of analytic functions with positive real part and a prescribed set of coefficients," Complex Variables and Elliptic Equations, vol. 17, no. 1-2, pp. 49-55, 1991. 
[7] Z. Peng, "The extreme points of a class of analytic functions with positive real part and a prescribed set of values," Acta Mathematica Scientia B, vol. 32, no. 5, pp. 1929-1936, 2012.

[8] D. J. Hallenbeck and T. H. MacGregor, "Support points of families of analytic functions described by subordination," Transactions of the American Mathematical Society, vol. 278, no. 2, pp. 523-546, 1983.

[9] Z. Peng, "The support points of several classes of analytic functions with fixed coefficients," Journal of Mathematical Analysis and Applications, vol. 340, no. 1, pp. 209-218, 2008.

[10] P. L. Duren, Univalent Functions, Springer, New York, NY, USA, 1983. 


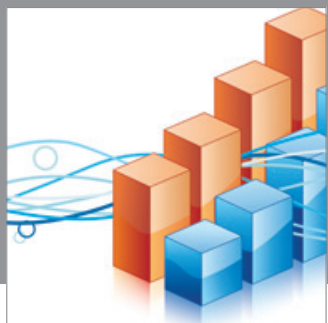

Advances in

Operations Research

mansans

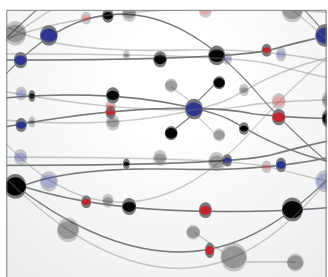

The Scientific World Journal
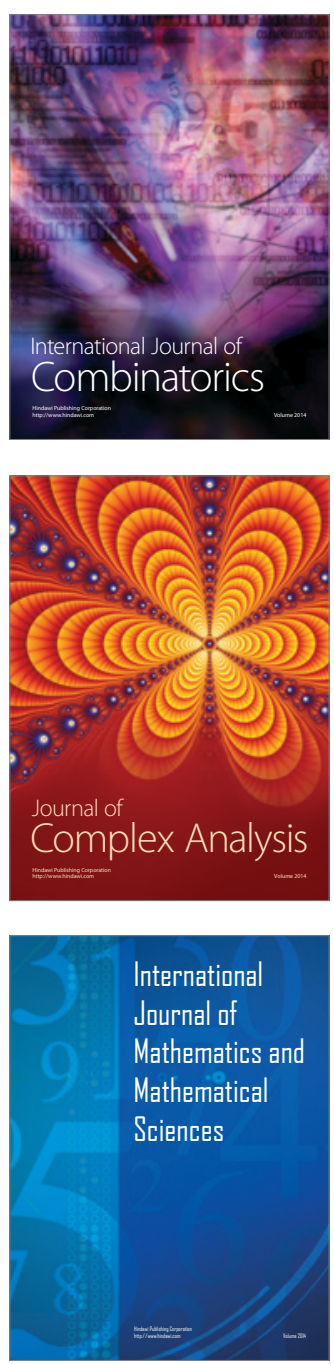
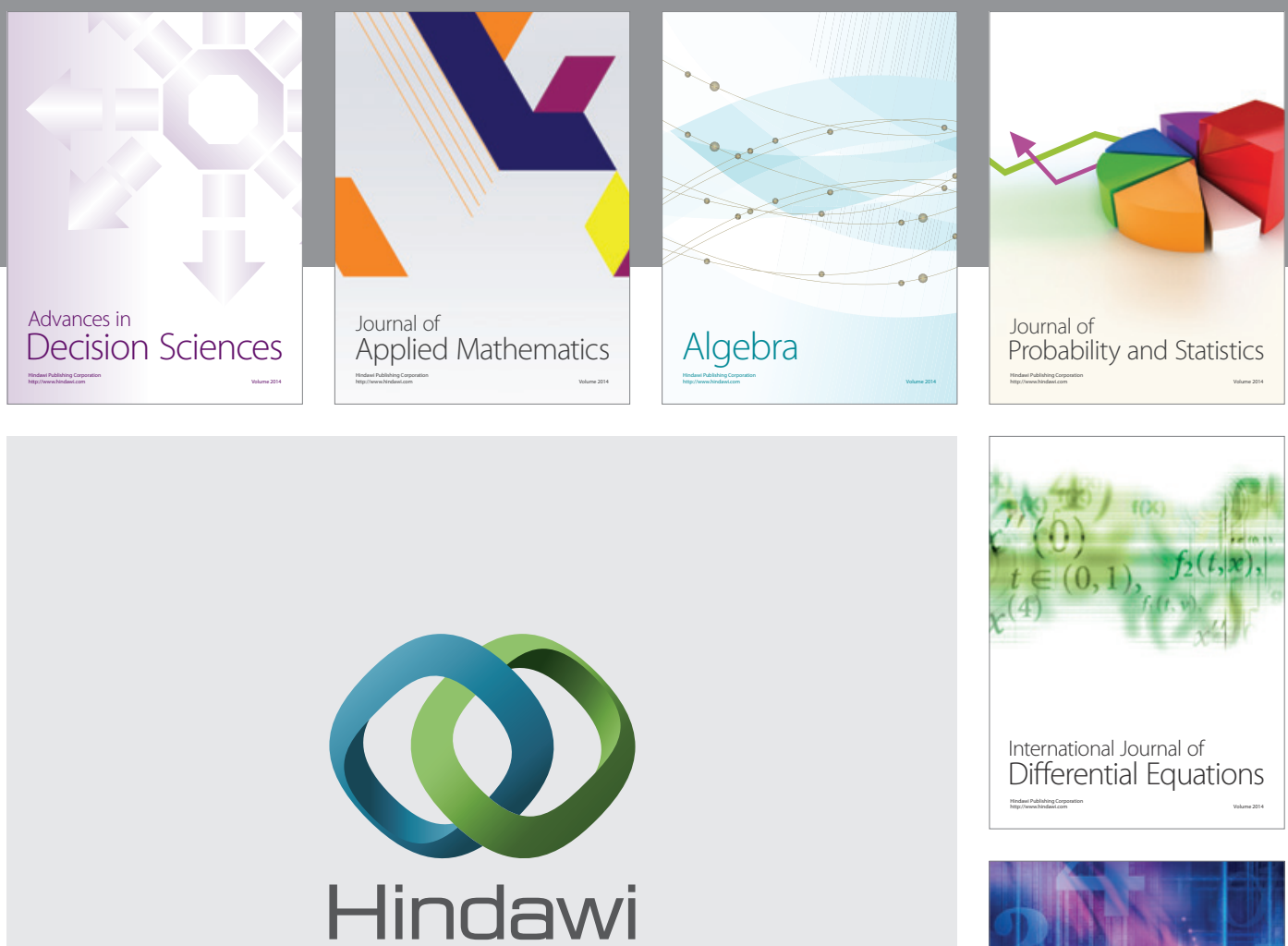

Submit your manuscripts at http://www.hindawi.com
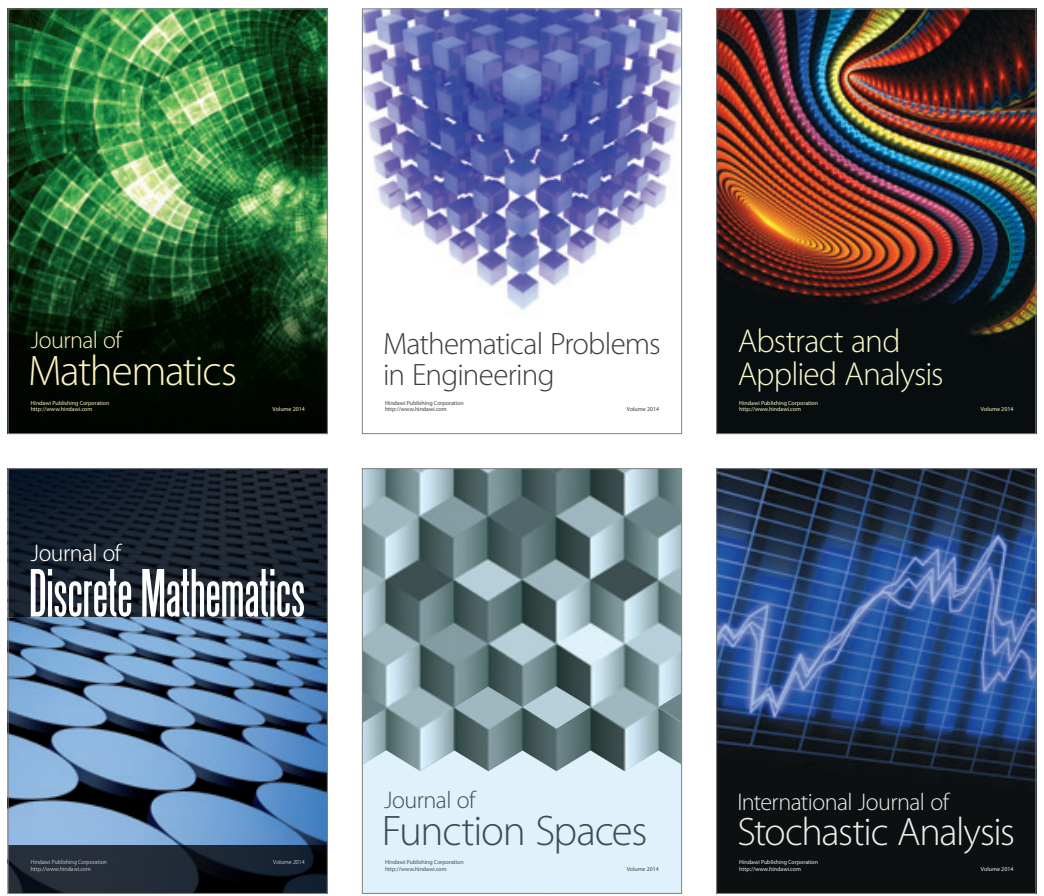

Journal of

Function Spaces

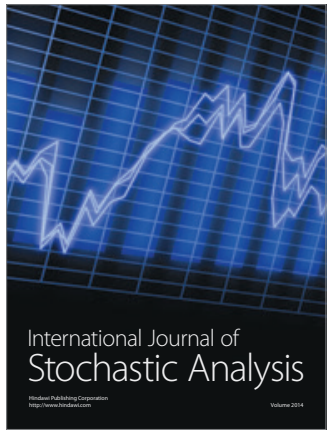

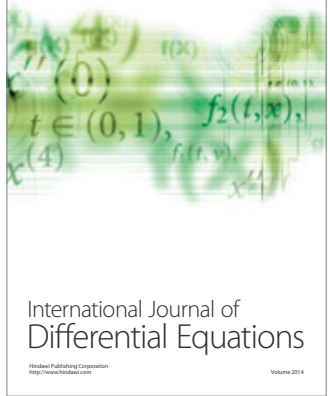
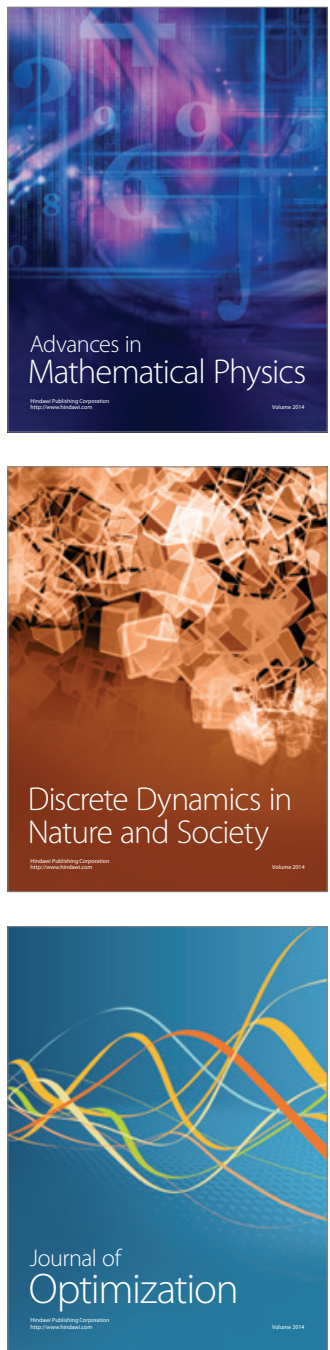